\title{
Composición y estructura de peces de la microcuenca del río Anchique, Tolima, Colombia
}

\author{
Diana Carolina Montoya-Ospina ${ }^{1}$, Francisco Antonio Villa-Navarro ${ }^{1}$ \\ \& Edwin Orlando López-Delgado ${ }^{1,2}$ \\ 1. Universidad del Tolima, Facultad de Ciencias, Departamento de Biología, Grupo de Investigación en Zoología, Barrio \\ Santa Elena, Ibagué, Tolima, Colombia; montoyadianac@gmail.com, favilla@ut.edu.co \\ 2. Estudiante Ph.D. Wildlife and Fisheries Science, Texas A \& M University, Winemiller Aquatic Ecology Lab., Texas, \\ USA; eolopezd@tamu.edu
}

Recibido 27-III-2017. Corregido 17-VIII-2017. Aceptado 20-IX-2017.

\begin{abstract}
Composition and structure of the fish community structure in the Anchique River sub-basin, Tolima, Colombia. The distribution and abundance of fish is influenced by multiple factors at temporal and spatial scales, and their community composition represent good river integrity indicators. The main objective of this study was to describe the composition and structure of fish assemblages along the Anchique river sub-basin (that drains to Magdalena river), and to test the hypothesis that these communities vary temporally and spatially. For this, four sites (E1, E2, E3 and E4) were sampled along the river (from river birthplace to Magdalena river), during the periods of high and low waters, in 2014. In each site, longitudinal transects (100 m long x $2 \mathrm{~m}$ wide, $60 \mathrm{~min})$ were established and fishing was made with both electrofishing and trawl $(10 \mathrm{~m} \times 1 \mathrm{~m}, 0.05 \mathrm{~m})$ nets. Fish samples were fixed and transported to the laboratory for posterior taxonomic identification with the use of keys; fish diversity and structure composition were calculated with the use of standard models. Besides, substrate composition and environmental variables were also considered. A total of 652 fish belonging to 36 species were recorded; high water showed higher values (325 fish with 32 species) than low water period (300 fish with 31 species). Siluriformes was dominant (51\%), followed by Characiformes (32\%), Gymnotiformes (5\%), Cyprinidontiformes and Cichliformes (6\%). Most abundant families were Characidae (28.3\%; 9 spp.), Loricariidae (23.4\%; 8 spp.) and Trichomycteridae (15.0\%; 2 spp.). Kruskal-Wallis test indicated that there is no significant difference among some environmental variables at the temporal level. The values of structural complexity and substrate composition indices showed that E4 had the highest values (0.8), followed by E1 (0.6), and E2 and E3 (0.4). Moreover, E4 had the highest richness and diversity, according to Hill's number. The canonical correspondence analysis showed that the substrate, and the complexity and width of the river channel, had a significant influence on fish composition and distribution. The fish community richness and diversity did not change temporarily, but did spatially, which could be related to the different structural features of sampling sites. Rev. Biol. Trop. 66(1): 336-351. Epub 2018 March 01.
\end{abstract}

Key words: ichthyofauna, aquatic ecosystem, environmental variables, Anchique river.

Las características estructurales de los ecosistemas acuáticos tropicales tienen influencia sobre la composición de las comunidades ícticas. La estructura de las comunidades ícticas en los ríos, se han relacionado principalmente con el tamaño del río, la velocidad, el caudal, la profundidad, la depredación y la competencia (Zaret \& Rand, 1971; Bussing \& López, 1977; Angermeier \& Karr, 1984; Greenberg,
1991; Harding, Burky, \& Way, 1998; Martin-Smith, 1998; Stewart, Ibarra, \& BarrigaSalazar, 2002), variables que cambian a nivel espacial y temporal.

Adicionalmente, la variabilidad temporal y la heterogeneidad del hábitat (como la composición del sustrato, ancho de la cobertura vegetal, complejidad estructural y la disponibilidad de nichos), influyen en los patrones 
de distribución de las comunidades de peces (Vannote, Minshall, Cummins, Sedell, \& Cushing, 1980); por tal razón, los hábitats de las comunidades ícticas son variables y espacialmente diversos (Frissell, Liss, Warren, \& Hurley, 1986).

Las comunidades de peces son, entonces, un indicador primario de la salud e integridad de un río, de tal forma que la declinación en la calidad de las comunidades es un indicador de una influencia humana negativa para el sistema (Mueller \& Pyron, 2010) como la disminución considerable del caudal debido a la creación de presas, o sequías producidas por la desviación del cauce para el riego de cultivos; o procesos físicos y biológicos que actúan como agentes de perturbación, como tormentas, inundaciones y sequías (Sousa, 1984). Para comprender estas comunidades es necesario conocer su abundancia y distribución, así como su relación con factores bióticos y abióticos que afectan sus cambios poblacionales (Oliveira \& Pessanha, 2014).

Algunas variables físicas de los ríos se relacionan directamente con parámetros fisicoquímicos, como ejemplo de ello, el caudal y el flujo del agua se asocian a la temperatura y $\mathrm{pH}$, las cuales pueden limitar la distribución y abundancia de las especies (Resh et al., 1988; Power, Sun, Parker, Dietrich, \& Wootton, 1995), y pueden fluctuar a nivel temporal y espacial.

En el departamento del Tolima los estudios ícticos han estado encaminados a evaluar aspectos de ecología reproductiva y trófica de las familias Trichomycteridae, Characidae, Sternopygidae, Cichlidae, Astroblepidae y Loricariidae en las microcuencas de los ríos de Coello y Prado (Villa-Navarro \& LosadaPrado, 1999; Villa-Navarro \& Losada-Prado, 2004; Briñez-Vásquez, Villa-Navarro, OrtegaLara, Reinoso-Flórez, \& García-Melo, 2005; García-Melo, Villa-Navarro, Reinoso-Flórez, \& Ortega-Lara, 2005; Zúñiga-Upegui, VillaNavarro, Ortega-Lara, \& Reinoso-Flórez, 2005), y en las microcuencas de los ríos Coello, Totare, Alvarado y Opia se han realizado estudios de diversidad, composición y estructura de las comunidades ícticas (CORTOLIMA, 2004; CORTOLIMA, 2007; López-Delgado, 2013; Albornoz-Garzón \& Conde-Saldaña, 2014); no obstante, en la microcuenca del río Anchique no se han realizado estudios ícticos. Por lo anterior el objetivo del presente estudio fue describir la estructura y composición de la comunidad íctica a lo largo del río Anchique y probar la hipótesis de que estas varían a nivel espacial y temporal.

\section{MATERIALES Y MÉTODOS}

Área de estudio: El estudio se desarrolló en la cuenca del río Anchique, el cual tributa directamente al río Magdalena, y se localiza en la zona de vida bosque seco tropical (bs-T), departamento de Tolima, Municipalidad de Natagaima, Colombia. Su cuenca drena un área de 25828.4 has y tiene una longitud de cauce de $47.4 \mathrm{~km}$.

Fase de campo: Se efectuaron dos muestreos en el 2014, uno en temporada de lluvias altas (febrero) y otro en lluvias bajas (abril). Se establecieron cuatro estaciones de muestreo a lo largo del cauce principal del río. La primera estación (E1) se ubicó cerca al nacimiento del río, a una altitud de $415 \mathrm{~m}$

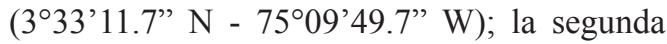
(E2) se ubicó a $346 \mathrm{~m}$ de altitud (3³3'56.3" $\mathrm{N}-75^{\circ} 08^{\prime} 43.3^{\prime \prime} \mathrm{W}$ ); la tercera (E3) se ubicó a $340 \mathrm{~m}\left(3^{\circ} 35^{\prime} 10.1^{\prime \prime} \mathrm{N}-75^{\circ} 07^{\prime} 54.2^{\prime \prime} \mathrm{W}\right)$; y la cuarta (E4) se ubicó a $3 \mathrm{~km}$ de su desembocadura en el río Magdalena, a $333 \mathrm{~m}$ de altitud $\left(3^{\circ} 34^{\prime} 28.7^{\prime}\right.$ ' N - 7505'59.1” W), la distancia entre E1 y E4 fue de 13 km (Fig. 1).

Las capturas se realizaron empleando un equipo de electropesca, en un transecto longitudinal de $100 \mathrm{~m}$ de largo y a $2 \mathrm{~m}$ de la orilla, durante $60 \mathrm{~min}$. Adicionalmente, entre dos personas fue utilizada una red de arrastre $(10 \mathrm{x}$ $1 \mathrm{~m}$, con un ojo de malla de $0.05 \mathrm{~m}$ ) con la cual se realizaron tres arrastres sobre las orillas en cada estación de muestreo, durante dos horas.

La electropesca es el método más adecuado para las condiciones que presentan los cuerpos de agua andinos, torrentosos y pedregosos 


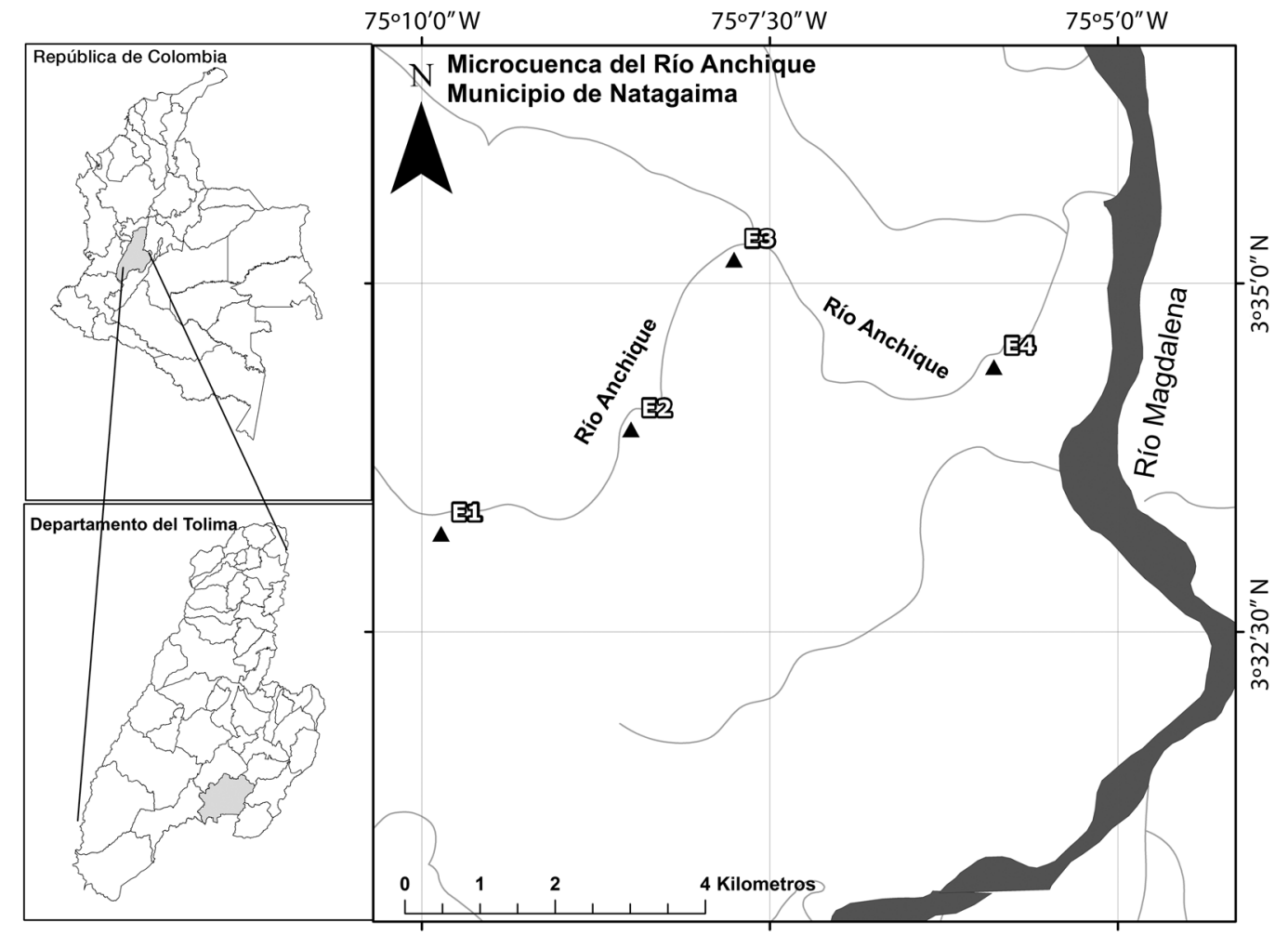

Fig. 1. Estaciones de muestreo en la microcuenca del río Anchique, Tolima, Colombia. Fig. 1. Location of the sampling sites in the Anchique River sub-basin, Tolima, Colombia.

(Maldonado-Ocampo et al., 2005); es ventajosa debido a que no es selectiva, y puede capturar peces de diferentes tamaños y no causa la muerte a los organismos, la electropesca está determinada principalmente por factores biológicos como la talla del pez, y por factores físicos como la conductividad y temperatura del agua (Guerrero-Kommritz, 1997).

Los peces capturados se fijaron en una solución de formol al $10 \%$, posteriormente fueron determinados taxonómicamente (Eigenmann, 1922; Dahl, 1971; Harold \& Vari, 1994; Buitrago, 1995; de Santana \& MaldonadoOcampo, 2005; Maldonado-Ocampo et al., 2005) en el Laboratorio de Investigación en Zoología de la Universidad del Tolima (GIZ). Una vez Identificados y cuantificados, los individuos fueron depositados en la Colección Zoológica sección Ictiología (CZUT-IC) de la
Universidad del Tolima; los individuos depositados en la Colección Zoológica van desde el lote CZUT-IC 11527 hasta 11915 en el primer muestreo, y CZUT-IC 11864 hasta 11929 en el segundo muestreo.

Paralelamente, se tomaron in situ algunos parámetros ambientales, tales como: temperatura superficial del agua empleando un termómetro de mercurio; velocidad $(\mathrm{m} / \mathrm{s})$ del agua con un flujómetro (General Oceanics - modelo 2030R y 2030R6); caudal ( $\left.\mathrm{m}^{3} / \mathrm{s}\right)$, profundidad (m) y ancho (m) del cauce. La profundidad media del agua en cada estación de muestreo fue calculada con base en medidas efectuadas con una varilla graduada, en intervalos de $1 \mathrm{~m}$ en cada una de las estaciones del río. Estas medidas sirvieron igualmente para calcular el ancho del río, posteriormente, el caudal del río, fue calculado utilizando el área y la velocidad. 
Adicionalmente, se colectaron muestras de agua superficial y contra corriente para su evaluación en el Laboratorio de Servicios de Extensión en análisis químico (LASEREX) de la Universidad del Tolima. Así, se determinó el $\mathrm{pH}$ (unidades de $\mathrm{pH})$, oxígeno disuelto $\left(\mathrm{mgO}_{2} /\right.$ $\mathrm{L})$, conductividad eléctrica ( $\mu \mathrm{S} / \mathrm{CM})$, alcalinidad $\left(\mathrm{HCO}_{3}^{-}\right)$, dureza $\left(\mathrm{mgCaCO}_{3} / \mathrm{L}\right)$ y sólidos totales $(\mathrm{mg} / \mathrm{L})$.

La toma de las muestras de agua se realizó siguiendo los procedimientos recomendados por el Órgano de Control Ambiental, cumpliendo con la normatividad vigente. Las muestras poseían la bitácora y los controles de calidad establecidos para la toma, rotulación, preservación, embalaje y entrega al laboratorio LASEREX acreditado ante el IDEAM, de acuerdo con lo establecido por el decreto 1600 del 27 de julio de 1994 expedido por el Ministerio de Ambiente y Desarrollo sostenible.

Variables ambientales: Para conocer la composición del sustrato y la complejidad estructural del hábitat fueron creadas dos matrices, las cuales fueron construidas teniendo en cuenta la presencia y ausencia de algunos componentes en cada estación de muestreo. Para la composición del sustrato se tuvo en cuenta la presencia y ausencia de roca, arena, hojarasca, rocas grandes, guijarro, grava y lodo; y, para la complejidad estructural del hábitat, se tuvo en cuenta la presencia y ausencia de hojarasca, vegetación enraizada sumergida, perifiton, vegetación riparia arbórea y arbustiva.

Posteriormente, se hallaron los índices de composición del sustrato (S) y complejidad estructural del hábitat (S), siguiendo la metodología propuesta por Winemiller, López-Fernández, Taphorn, Nico and Duque (2008), los cuales estandarizaron el índice (S) por medio de la siguiente fórmula:

$$
\mathrm{S}=\frac{\sum x_{i}}{N}
$$

Donde, S son los índices de composición del sustrato y complejidad estructural del hábitat, $\mathrm{x}_{\mathrm{i}}$ representa cada uno de los componentes del sustrato y estructura del hábitat por cada estación de muestreo, y $\mathrm{N}$ representa el número de componentes combinados del sustrato o estructura del hábitat observados en las estaciones de muestreo; los valores cercanos a 0 indican mínima complejidad y cercanos a 1 máxima complejidad (Winemiller et al., 2008).

Para determinar la existencia de diferencias estadísticamente significativas entre variables físicas y físicoquímicas de las estaciones de muestreo a nivel espacial y temporal, se realizó una prueba no paramétrica de una vía de Kruskal-Wallis. Se realizó esta prueba debido a que los datos no cumplieron los supuestos de normalidad y homogeneidad de varianza, estos fueron evaluados con la prueba de ShapiroWilk y la prueba de Bartlett respectivamente. Se utilizó la función Kruskal test de la librería Stats del programa estadístico $\mathrm{R}$ versión 3.2.5 (R Development Core Team, 2016).

Con el objetivo de identificar el régimen pluviométrico en la zona de estudio, fueron utilizados los datos de precipitaciones anuales y mensuales de los últimos diez años, con el fin de realizar un diagrama de precipitación con la media mensual multianual, y la media mensual del año de estudio (2014). Estos datos fueron obtenidos de estaciones pluviométricas cercanas a la zona de estudio del Instituto de Hidrología, Meteorología y Estudios Ambientales de Colombia (IDEAM).

Estructura y composición: Para determinar la representatividad de los muestreos, se calcularon los estimadores de diversidad Chao 1, ACE y Cole, para esto se utilizó el paquete estadístico EstimateS versión 8.2 (Colwell, 2009).

La abundancia relativa se calculó a partir del número de individuos colectados de cada especie y su relación con el número total de individuos de la muestra. Se calculó el Índice de Constancia (C), el cual indica que las especies presentes en más del $50 \%$ de las muestras pueden ser consideradas como residentes, entre el 25 y $50 \%$ son accesorias y, menos del $25 \%$ son accidentales (Ferreira, 2007). 
Además, fueron calculados los modelos de distribución de abundancia los cuales incorporan información sobre la riqueza, la distribución y, en algunas ocasiones, el número de especies no observadas en una comunidad (McGill et al., 2007). Estos son una herramienta útil para describir la estructura de la comunidad y pueden ser indicadores de algún tipo de disturbio (Hill \& Hamer, 1998).

Para determinar la estructura de la comunidad íctica, se evaluaron las abundancias proporcionales de cada una de las especies, desarrollando los modelos serie geométrica, logarítmica, log-normal y vara quebrada de MacArthur (Moreno, 2001; Magurran, 2004); se empleó la prueba de bondad de ajuste Kolmogorov-Smirnov (Magurran, 2004) para establecer cuál de ellos explica mejor la distribución de los datos.

Para medir la diversidad a nivel espacial, se hallaron los números efectivos de especies o números de Hill, a través de la ecuación denominada ${ }^{\mathrm{q}} \mathrm{D}$ (Jost, 2006).

$$
{ }^{\mathrm{q}} \mathrm{D}=\left(\sum_{i=1}^{S} P_{i}^{q}\right)^{1 /(1-\mathrm{q})}
$$

Dónde ${ }^{\mathrm{q}} \mathrm{D}$ es la diversidad. El exponente $q$ determina la sensibilidad del índice a las abundancias relativas de las especies. Tiene tres componentes, la diversidad de orden cero $(q=$ 0 ) el cual es insensible a las abundancias de las especies, el valor obtenido es el equivalente a la riqueza de especies; la diversidad de orden 1 $(q=1)$ incluye a todas las especies con un peso exactamente proporcional a su abundancia en la comunidad, y tiene en cuenta las especies comunes; y, los valores de $q$ mayores a 1 ( $q$ $=2$ ), tienen más en cuenta las especies dominantes (Hill, 1973). Todo lo anterior se realizó haciendo uso del programa estadístico $\mathrm{R}$ versión 3.2.5 y la librería Vegan (R Development Core Team, 2016).

Asimismo, se realizó un análisis de rarefacción y extrapolación usando el número efectivo de especies, mediante los órdenes de diversidad $q=0, q=1$ y $q=2$ (números de Hill), con el fin de identificar diferencias en la diversidad de especies a nivel temporal y espacial (Chao et al., 2014), usando el programa estadístico R versión 3.2.5 y la librería iNEXT. (R Development Core Team, 2016).

Para definir si la composición de la comunidad de peces es similar dentro de cada estación de muestreo, se realizó un análisis de escalamiento multidimensional no métrico (EMNM), usando la distancia de Bray-Curtis; el análisis se consideró robusto cuando el valor de estrés fue menor a 0.2 . Se utilizó programa estadístico $\mathrm{R}$ versión 3.2.5 y la librería Vegan (R Development Core Team, 2016). Posterior al ordenamiento, se realizó un análisis de similitud (ANOSIM) (Chapman \& Underwood, 1999) con el fin de determinar diferencias significativas entre dos o más grupos. Para este caso, se determinaron grupos a nivel espacial y temporal. Este análisis se desarrolló usando la librería Vegan del programa estadístico $\mathrm{R}$ versión 3.2.5 (R Development Core Team, 2016).

Influencia de los factores ambientales: Con el fin de determinar si las variables ambientales (incluyendo los valores del índice de composición del sustrato y complejidad estructural del hábitat) influyeron en la estructura de la comunidad de peces, se realizó un análisis de ordenación. El método utilizado fue el de correspondencia canónica (ACC) el cual es ampliamente utilizado para matrices que poseen datos de abundancia ya que este método utiliza la distancia de $X^{2}$ (Chi-cuadrado).

Después de realizar el análisis de ordenación, se procedió a realizar la selección de las variables, con el fin de determinar cuál de estas influyó de manera significativa en la estructura de la comunidad. Para esto, fue realizado un análisis de permutación de Monte Carlo (999 permutaciones). El ACC y la prueba de Monte Carlo fueron realizados utilizando el programa CANOCO 4.5 (Braa, Ter, \& Smilauer, 2009).

\section{RESULTADOS}

Variables ambientales: La caracterización física de las estaciones en altas lluvias mostró que el cauce fue más ancho en E2 (20 m) y menos ancho en E1 (4 m); el caudal osciló 
entre $2.35 \mathrm{~m}^{3} / \mathrm{s}(\mathrm{E} 2)$ y $0.43 \mathrm{~m}^{3} / \mathrm{s}(\mathrm{E} 4)$; la profundidad fue mayor en E1 $(0.37 \mathrm{~m})$ y menor en E4 $(0.23 \mathrm{~m})$; y finalmente, la velocidad fue mayor en E3 y E4 (0.377 m / s) (Cuadro 1). En bajas lluvias, el ancho fue mayor en E2 (18.8 m) y menor en E1 $(4.1 \mathrm{~m})$; el caudal osciló entre $3.86 \mathrm{~m}^{3} / \mathrm{s}$ (E2) y $0.65 \mathrm{~m}^{3} / \mathrm{s}$ (E1); la profundidad fue mayor en E1 $(0.52 \mathrm{~m}) \mathrm{y}$ menor en E4 $(0.18 \mathrm{~m})$; y finalmente, la velocidad fue mayor en E2 y E3 $(0.69 \mathrm{~m} / \mathrm{s})$ y menor en E1 $(0.30 \mathrm{~m} / \mathrm{s})$. No se encontraron diferencias estadísticamente significativas entre las variables físicas del río entre periodos climáticos (Kruskal-Wallis, profundidad $\mathrm{p}=0.08326$; ancho $\mathrm{p}=0.1489$; velocidad $\mathrm{p}=0.2454$; caudal $\mathrm{p}=1$ ).

Respecto a la caracterización fisicoquímica, en altas lluvias E1 presentó los valores más altos de $\mathrm{pH}$ (7.6), dureza (124.2 $\mathrm{mg} \mathrm{CaCO}_{3} /$ L) y sólidos totales (268); contrario a esto, los valores más bajos de estas variables se hallaron en E2. La temperatura del agua fue mayor en E4 $\left(30{ }^{\circ} \mathrm{C}\right)$ y menor en E1 $\left(24^{\circ} \mathrm{C}\right)$. En bajas lluvias, los valores de las variables de $\mathrm{pH}$ (7.6), dureza (111.8 $\mathrm{mg} \mathrm{CaCO}_{3} / \mathrm{L}$ ), conductividad eléctrica $(388 \mu \mathrm{s} / \mathrm{cm})$ y sólidos totales (300) fueron también mayores en E1. El oxígeno disuelto osciló entre $6.33 \mathrm{mg} \mathrm{O}_{2} / \mathrm{L}$ (E4) y $5.55 \mathrm{mgO}_{2} / \mathrm{L}(\mathrm{E} 1)$; la temperatura del agua entre $30{ }^{\circ} \mathrm{C}\left(\mathrm{E} 3\right.$ y E4) y $26{ }^{\circ} \mathrm{C}(\mathrm{E} 1)$. No se encontraron diferencias estadísticamente significativas entre las variables fisicoquímicas del río entre periodos climáticos (Kruskal-Wallis, $\mathrm{pH} \mathrm{p}=0.7728$; dureza $\mathrm{p}=0.7728$; alcalinidad $\mathrm{p}=0.5637$, conductividad eléctrica $\mathrm{p}=0.6631$, oxígeno disuelto $\mathrm{p}=0.08326$; sólidos totales $\mathrm{p}$ $=0.1465$ y temperatura del agua $\mathrm{p}=0.3719$ ).

A nivel general, el cálculo del índice complejidad estructural del hábitat (S) indicó que E4 presentó el mayor valor (0.8), seguida de E1 (0.6), mientras que E2 y E3 fueron las menos complejas (0.4); en cuanto al índice de composición del sustrato E4 presentó el mayor valor (0.72), seguida de E2 (0.43) y E3 (0.43), y finalmente, E1 obtuvo el valor más bajo (0.29).

Estructura y composición: La curva de acumulación de especies indica que el número total de especies observadas $($ Smean $=36)$ en el río Anchique corresponde al $99.31 \%$ del valor esperado por el estimador Chao 1, 98.46\% para ACE y $100 \%$ para Cole, indicando una buena representatividad.

Se recolectaron un total de 625 individuos, pertenecientes a cinco órdenes, 17 familias, 31 géneros y 36 especies. En lluvias altas se recolectaron 325 pertenecientes a 32 especies, mientras que para lluvias bajas se recolectaron

CUADRO 1

Variables físicas y químicas por estación de muestreo de la microcuenca del río Anchique, Tolima, Colombia

TABLE 1

Physical and chemical variables by sampling sites in the Anchique river sub-basin

\begin{tabular}{lcccccccc} 
& \multicolumn{2}{c}{ Estación 1 } & \multicolumn{2}{c}{ Estación 2} & \multicolumn{2}{c}{ Estación 3 } & \multicolumn{2}{c}{ Estación 4} \\
Variables físicas y químicas & Altas lluvias & Bajas lluvias & Altas lluvias & Bajas lluvias & Altas lluvias & Bajas lluvias Altas lluvias Bajas lluvias \\
Profundidad (m) & 0.372 & 0.52 & 0.33 & 4.12 & 0.24 & 0.3 & 0.23 & 0.65 \\
Ancho $(\mathrm{m})$ & 4 & 0.29 & 20 & 18.8 & 14 & 0.69 & 5 & 3.86 \\
Velocidad (m / s) & 0.3 & 0.26 & 0.35 & 8 & 0.37 & 0.69 & 0.37 & 1.46 \\
Caudal $\left(\mathrm{m}^{3} / \mathrm{s}\right)$ & 0.45 & 0.18 & 2.35 & 4.1 & 1.27 & 0.82 & 0.43 & 1.05 \\
$\mathrm{pH}$ & 7.62 & 7.68 & 7.15 & 7.44 & 7.46 & 7.12 & 7.26 & 7.56 \\
Dureza $\left(\mathrm{mg} \mathrm{CaCO}_{3} / \mathrm{L}\right)$ & 124.2 & 111.8 & 1 & 3 & 4.4 & 0.9 & 6.1 & 6.4 \\
Alcalinidad $\mathrm{HCO}^{3-}$ & 182 & 162.4 & 42 & 34.6 & 41 & 40.3 & 45 & 48.1 \\
Conductividad eléctrica & 379 & 388 & 82.5 & 75.4 & 87.8 & 78.2 & 89.6 & 89.6 \\
Oxígeno disuelto & 6.3 & 5.55 & 6.85 & 6.29 & 7.57 & 6.36 & 7.59 & 6.33 \\
Sólidos totales & 268 & 300 & 86 & 182 & 80 & 161 & 80 & 154 \\
Temperatura del agua $\left({ }^{\circ} \mathrm{C}\right)$ & 24 & 26 & 25 & 26 & 28 & 30 & 30 & 30 \\
\hline
\end{tabular}


e identificaron 300 individuos pertenecientes a 31 especies (Cuadro 2).

En la cuenca del río Anchique el orden Siluriformes fue el más dominante $(51 \%)$, seguido de Characiformes (32\%). Gymnotiformes, Cyprinidontiformes y Cichliforrmes tuvieron una representación entre el $5 \%$ y $6 \%$. A nivel de familias, las más abundantes fueron Characidae (28.3\%; 9 spp.), Loricariidae (23.4 $\%$; 8 spp.) y Trichomycteridae (15.0\%; 2 spp.). Las especies más abundantes fueron Creagrutus affinis (16.3\%), Chaetostoma milesi (14.4 \%) y Trichomycterus banneaui (13.1 $\%$ ), que representaron más del $42.8 \%$ de la abundancia total.

Para las dos épocas muestreadas, los órdenes con mayor abundancia relativa fueron Siluriformes y Characiformes que aportaron el $80 \%$ de la abundancia, aproximadamente. En cuanto a familias, Characidae y Loricariidae aportaron cerca del $55 \%$ de la abundancia en lluvias altas y bajas.
En lluvias altas, Chaetostoma milesi (20 \%), Trichomycterus banneaui (16.9\%) y Creagrutus affinis (13.2\%) fueron las especies más abundantes; mientras que Leporinus muyscorum, Hoplias malabaricus, Gasteropelecus maculatus, Rhamdia guatemalensis y Chaetostoma thomsoni sólo se registraron en este periodo. Durante lluvias bajas, C. affinis (19.6 $\%)$ y Chaetostoma sp. (11.6\%) fueron las especies más abundantes; Astyanax magdalenae, Chaetostoma sp. y Lasiancistrus caucanus únicamente se registraron en esta temporada.

En general, el Índice de Constancia muestra a Astyanax fasciatus, C. affinis, T. banneaui y Apteronotus eschemeyeri como residentes $(\mathrm{C}=11.1 \%)$, mientras que $A$. magdalenae, Leporinus muyscorum, Hoplias malabaricus, Gasteropelecus maculatus, Chaetostoma thomsoni y Kronoheros umbrifer $(\mathrm{C}=16.7 \%)$ se consideran accidentales, y las 26 especies restantes fueron accesorias para el río (C $=72.2 \%)$ (Cuadro 2).

CUADRO 2

Lista de especies ícticas por estaciones de muestreo y época climática e Índice de Constancia de la microcuenca del río Anchique

TABLE 2

Fish species by sampling stations, climatic period and Constancy Index of the Anchique river sub-basin

\begin{tabular}{|c|c|c|c|c|c|c|c|c|c|}
\hline \multirow[b]{2}{*}{ Taxón } & \multicolumn{2}{|c|}{ Estación 1} & \multicolumn{2}{|c|}{ Estación 2} & \multicolumn{2}{|c|}{ Estación 3} & \multicolumn{2}{|c|}{ Estación 4} & \multirow[b]{2}{*}{ Índice de constancia } \\
\hline & $\begin{array}{c}\text { Altas } \\
\text { lluvias }\end{array}$ & $\begin{array}{c}\text { Bajas } \\
\text { lluvias }\end{array}$ & $\begin{array}{c}\text { Altas } \\
\text { lluvias }\end{array}$ & $\begin{array}{l}\text { Bajas } \\
\text { lluvias }\end{array}$ & $\begin{array}{c}\text { Altas } \\
\text { lluvias }\end{array}$ & $\begin{array}{l}\text { Bajas } \\
\text { lluvias }\end{array}$ & $\begin{array}{c}\text { Altas } \\
\text { lluvias }\end{array}$ & $\begin{array}{c}\text { Bajas } \\
\text { lluvias }\end{array}$ & \\
\hline \multicolumn{10}{|l|}{ Characiformes } \\
\hline \multicolumn{10}{|l|}{ Parodontidae } \\
\hline Parodon magdalenesis & 0 & 0 & 0 & 0 & 5 & 1 & 0 & 0 & Accesoria \\
\hline \multicolumn{10}{|l|}{ Crenuchidae } \\
\hline Characidium phoxocephalum & 8 & 3 & 0 & 0 & 2 & 0 & 0 & 0 & Accesoria \\
\hline \multicolumn{10}{|l|}{ Characidae } \\
\hline Argopleura magdalenensis & 0 & 0 & 4 & 2 & 0 & 0 & 0 & 1 & Accesoria \\
\hline Astyanax fasciatus & 0 & 0 & 1 & 3 & 5 & 3 & 2 & 15 & Residente \\
\hline Astyanax magdalenae & & 0 & & 0 & & 0 & & 2 & Accidental \\
\hline Creagrutus affinis & 0 & 15 & 3 & 0 & 37 & 29 & 3 & 15 & Residente \\
\hline Gephyrocharax melanocheir & 0 & 0 & 1 & 0 & 0 & 0 & 0 & 5 & Accesoria \\
\hline Hemibrycon raqueliae & 0 & 0 & 0 & 1 & 1 & 0 & 0 & 2 & Accesoria \\
\hline Microgenys minuta & 0 & 0 & 1 & 6 & 8 & 7 & 0 & 0 & Accesoria \\
\hline Saccoderma hastata & 0 & 0 & 0 & 0 & 0 & 0 & 1 & 5 & Accesoria \\
\hline \multicolumn{10}{|l|}{ Anostomidae } \\
\hline Leporinus muyscorum & 0 & 0 & 0 & 0 & 0 & 0 & 2 & 0 & Accidental \\
\hline Erithrinidae & & & & & & & & & \\
\hline
\end{tabular}


CUADRO 2 (Continuación) / TABLE 2 (Continued)

\begin{tabular}{|c|c|c|c|c|c|c|c|c|c|}
\hline \multirow[b]{2}{*}{ Taxón } & \multicolumn{2}{|c|}{ Estación 1} & \multicolumn{2}{|c|}{ Estación 2} & \multicolumn{2}{|c|}{ Estación 3} & \multicolumn{2}{|c|}{ Estación 4} & \multirow[b]{2}{*}{ Índice de constancia } \\
\hline & $\begin{array}{l}\text { Altas } \\
\text { lluvias }\end{array}$ & $\begin{array}{l}\text { Bajas } \\
\text { lluvias }\end{array}$ & $\begin{array}{c}\text { Altas } \\
\text { lluvias }\end{array}$ & $\begin{array}{c}\text { Bajas } \\
\text { lluvias }\end{array}$ & $\begin{array}{c}\text { Altas } \\
\text { lluvias }\end{array}$ & $\begin{array}{l}\text { Bajas } \\
\text { lluvias }\end{array}$ & $\begin{array}{l}\text { Altas } \\
\text { lluvias }\end{array}$ & $\begin{array}{l}\text { Bajas } \\
\text { lluvias }\end{array}$ & \\
\hline Hoplias malabaricus & 0 & 0 & 0 & 0 & 0 & 0 & 2 & 0 & Accidental \\
\hline \multicolumn{10}{|l|}{ Gasteropelecidae } \\
\hline Gasteropelecus maculatus & 0 & 0 & 0 & 0 & 0 & 0 & 2 & 0 & Accidental \\
\hline \multicolumn{10}{|l|}{ Siluriformes } \\
\hline \multicolumn{10}{|l|}{ Pimelodidae } \\
\hline Pimelodus blochii & 0 & 0 & 0 & 0 & 0 & 0 & 12 & 2 & Accesoria \\
\hline Rhamdia guatemalensis & 0 & 0 & 0 & 0 & 2 & 0 & 1 & 0 & Accesoria \\
\hline \multicolumn{10}{|l|}{ Heptapteridae } \\
\hline Cetopsorhamdia molinae & 0 & 0 & 0 & 1 & 16 & 11 & 0 & 0 & Accesoria \\
\hline Pimelodella chagresi & 0 & 0 & 0 & 0 & 3 & 1 & 0 & 0 & Accesoria \\
\hline Heptapteridae sp. & 0 & 0 & 0 & 0 & 8 & 9 & 0 & 1 & Accesoria \\
\hline \multicolumn{10}{|l|}{ Cetopsidae } \\
\hline Cetopsis othonops & 0 & 0 & 0 & 2 & 1 & 2 & 0 & 0 & Accesoria \\
\hline \multicolumn{10}{|l|}{ Trichomycteridae } \\
\hline Trichomycterus retropinnis & 4 & 8 & 0 & 0 & 0 & 0 & 0 & 0 & Accesoria \\
\hline Trichomycterus banneaui & 4 & 5 & 8 & 7 & 43 & 14 & 0 & 1 & Residente \\
\hline \multicolumn{10}{|l|}{ Loricariidae } \\
\hline Chaetostoma milesi & 0 & 0 & 23 & 25 & 42 & 0 & 0 & 0 & Accesoria \\
\hline Chaetostoma thomsoni & 0 & 0 & 0 & 0 & 1 & 0 & 0 & 0 & Accidental \\
\hline Chaetostoma sp. & 0 & 0 & 0 & 0 & 0 & 34 & 0 & 1 & Accesoria \\
\hline Lasiancistrus caucanus & 0 & 0 & 0 & 2 & 0 & 2 & 0 & 1 & Accesoria \\
\hline Sturisomatichthys leightoni & 0 & 0 & 0 & 0 & 6 & 1 & 0 & 2 & Accesoria \\
\hline Spatuloricaria gymnogaster & 0 & 0 & 0 & 0 & 2 & 3 & 0 & 1 & Accesoria \\
\hline \multicolumn{10}{|l|}{ Astroblepidae } \\
\hline Astroblepus homodon & 1 & 6 & 0 & 0 & 0 & 0 & 0 & 0 & Accesoria \\
\hline \multicolumn{10}{|l|}{ Gymnotiformes } \\
\hline \multicolumn{10}{|l|}{ Sternopygidae } \\
\hline Eigenmaniia virescens & 0 & 0 & 0 & 0 & 0 & 0 & 7 & 4 & Accesoria \\
\hline Sternopygus aequilabiatus & 0 & 0 & 0 & 0 & 1 & 0 & 0 & 1 & Accesoria \\
\hline \multicolumn{10}{|l|}{ Apteronotidae } \\
\hline Apteronotus eschmeyeri & 0 & 0 & 1 & 5 & 7 & 2 & 7 & 3 & Residente \\
\hline \multicolumn{10}{|l|}{ Cyprinodontiformes } \\
\hline \multicolumn{10}{|l|}{ Cynolebiidae } \\
\hline Cynodonichthys magdalenae & 16 & 13 & 0 & 0 & 0 & 0 & 0 & 0 & Accesoria \\
\hline \multicolumn{10}{|l|}{ Poeciliidae } \\
\hline Poecilia caucana & 0 & 0 & 0 & 0 & 0 & 0 & 2 & 5 & Accesoria \\
\hline \multicolumn{10}{|l|}{ Cichliformes } \\
\hline \multicolumn{10}{|l|}{ Cichlidae } \\
\hline Andinoacara latifrons & 0 & 0 & 0 & 0 & 0 & 0 & 4 & 5 & Accesoria \\
\hline Kronoheros umbrifer & 0 & 0 & 0 & 0 & 0 & 0 & 0 & 1 & Accidental \\
\hline Geophagus steindachneri & 15 & 5 & 0 & 0 & 0 & 0 & 0 & 0 & Accesoria \\
\hline
\end{tabular}


En altas lluvias las especies residentes no variaron $(\mathrm{C}=12.5 \%)$, pero el número de especies accesorias aumentó a $28(\mathrm{C}=87.5 \%)$; en bajas lluvias nuevamente se registraron las mismas especies residentes, pero se adicionó L. caucanus (16.1\%) y bajó a 26 el número de accesorias $(\mathrm{C}=83.8 \%)$.

La distribución de especies del río Anchique, a nivel general, se ajustó al modelo de serie logarítmica (D36 $=0.2194 ; \mathrm{p}>0.05)$, el cual no varió entre las épocas evaluadas (altas lluvias, D32 $=0.0812 ; \mathrm{p}>0.05$ ) (bajas lluvias, $\mathrm{D} 31=0.2419 ; \mathrm{p}>0.05)$.

A partir de la extrapolación del número de especies $\left({ }^{0} \mathrm{D}\right)$ a nivel espacial, se determinó que E4 (24 spp.) y E3 (20 spp.) concentran la mayor riqueza; en contraste, E2 (12 spp.) y E1 (7 spp.) fueron las estaciones con menor riqueza de especies.

A partir del número de especies típicas o comunes $\left({ }^{1} \mathrm{D}\right)$ se evidenció que E4 (14 spp.) y E3 (10 spp.) obtuvieron los mayores valores, y los más bajos se observan en E1 (6 spp.) y E2 (5 spp.). Con respecto al número de especies dominantes $\left({ }^{2} \mathrm{D}\right), \mathrm{E} 4$ (11 spp.) y E3 (8 spp.) obtuvieron los valores más altos, y finalmente, los valores más bajos se observan en E1 (5 spp.) y E2 (3 spp.).

Finalmente, el número efectivo de especies en los órdenes $0(q=0), 1(q=1)$ y $2(q=2)$ no mostraron diferencias entre las épocas de altas y bajas lluvias evaluadas.

El análisis EMNM (stress $=0.04)$ muestra que la composición de la comunidad fue diferente en E1 y E4 (Fig. 2). La comunidad íctica en E1 estuvo conformado por Characidium phoxocephalum, C. affinis, Trichomycterus retropinnis, T. banneaui, Astroblepus homodon, Cynodonichthys magdalenae y Geophagus steindachneri; compartiendo a C. affinis y $T$. banneaui con las demás estaciones, y $C$. phoxocephalum con E3.

En la estación E4 se registró el mayor número de especies, mientras que en la estación E1 se presentó el menor número de especies. En este orden, la comunidad de la estación E4 se caracterizó por la presencia de A. magdalenae, Saccoderma hastata, L. muyscorum,
H. malabaricus, G. maculatus, Pimelodus blochii, Eigenmannia virescens, Poecilia caucana, Andinoacara latifrons y C. umbrifera, estas especies se registraron únicamente en $\mathrm{E} 4$.

Las 12 especies presentes en E2 son compartidas con una $\mathrm{u}$ otra estación, mientras que E3, con 20 especies, tiene a Parodon magdalenensis, Pimelodella chagresi y Chaetostoma thomsoni como especies no compartidas con ninguna otra estación. Finalmente, se detectaron diferencias en las comunidades de peces espacialmente (ANOSIM, $\mathrm{p}<0.012$ ), no siendo así entre periodos climáticos (ANOSIM, $\mathrm{p}>0.742$ ).

Influencia de los factores ambientales: El análisis de correspondencia canónica, indicó que el componente 1 explicó el $33 \%$ de la variación de las especies y el componente 2 un 27 \%. El ACC y la prueba de Monte Carlo (999 permutaciones), muestran que la composición del sustrato ( $p=0.022 ; \mathrm{F}=2.39$ ), la complejidad estructural del hábitat $(\mathrm{p}=0.012 ; \mathrm{F}=$ $3.20)$ y el ancho del cauce $(\mathrm{p}=0.052 ; \mathrm{F}=2.39)$

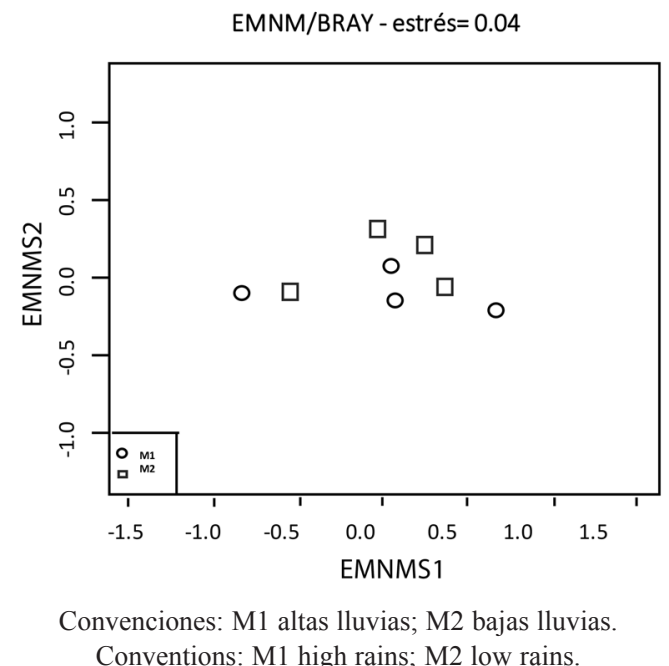

Fig. 2. Escalamiento Multidimensional No Métrico $($ EMNM, estrés $=0.04)$ de la composición de la comunidad íctica en las dos épocas de muestreo evaluadas a lo largo de la microcuenca del río Anchique.

Fig. 2. Non-Metric Multidimensional Scaling (NMDS) of the fish fauna composition in the two seasons evaluate along the Anchique river sub-basin. 
influyeron significativamente en la composición y distribución de la fauna íctica; mientras que las variables fisicoquímicas no tuvieron ninguna influencia en la composición y distribución de la fauna a lo largo del río Anchique.

La composición del sustrato y la complejidad estructural del hábitat fueron los factores que más influyeron en la comunidad de E4, en los dos periodos climáticos, asociándose con A. magdalenae, G. melanocheir, S. hastata, L. muyscorum, H. malabaricus, G. maculatus, P. blochii, R. guatemalensis, Chaetostoma sp., S. leightoni, E. virescens, S. aequilabiatus, P. caucana, A. latifrons, A. eschmeyeri y K. umbrifer (Fig. 3).
El caudal y ancho del cauce determinaron la comunidad de peces en E2 y E3, donde se registran a $A$. magdalenensis, A. fasciatus, $C$. affinis, $H$. raqueliae, M. minuta, C. molinae, $C$. othonops, P. magdalenesis, S. gymnogaster, $P$. chagresi, T. banneaui, Ch. milesi y L. caucanus.

El diagrama de ordenación del ACC concuerda con el diagrama de ordenación del EMNM, en que las estaciones más similares por composición de especies, estructura de la comunidad y variables ambientales son E2 y E3 (Fig. 3), ya que se encuentran agrupadas en el mismo cuadrante del diagrama y comparten un número similar especies.

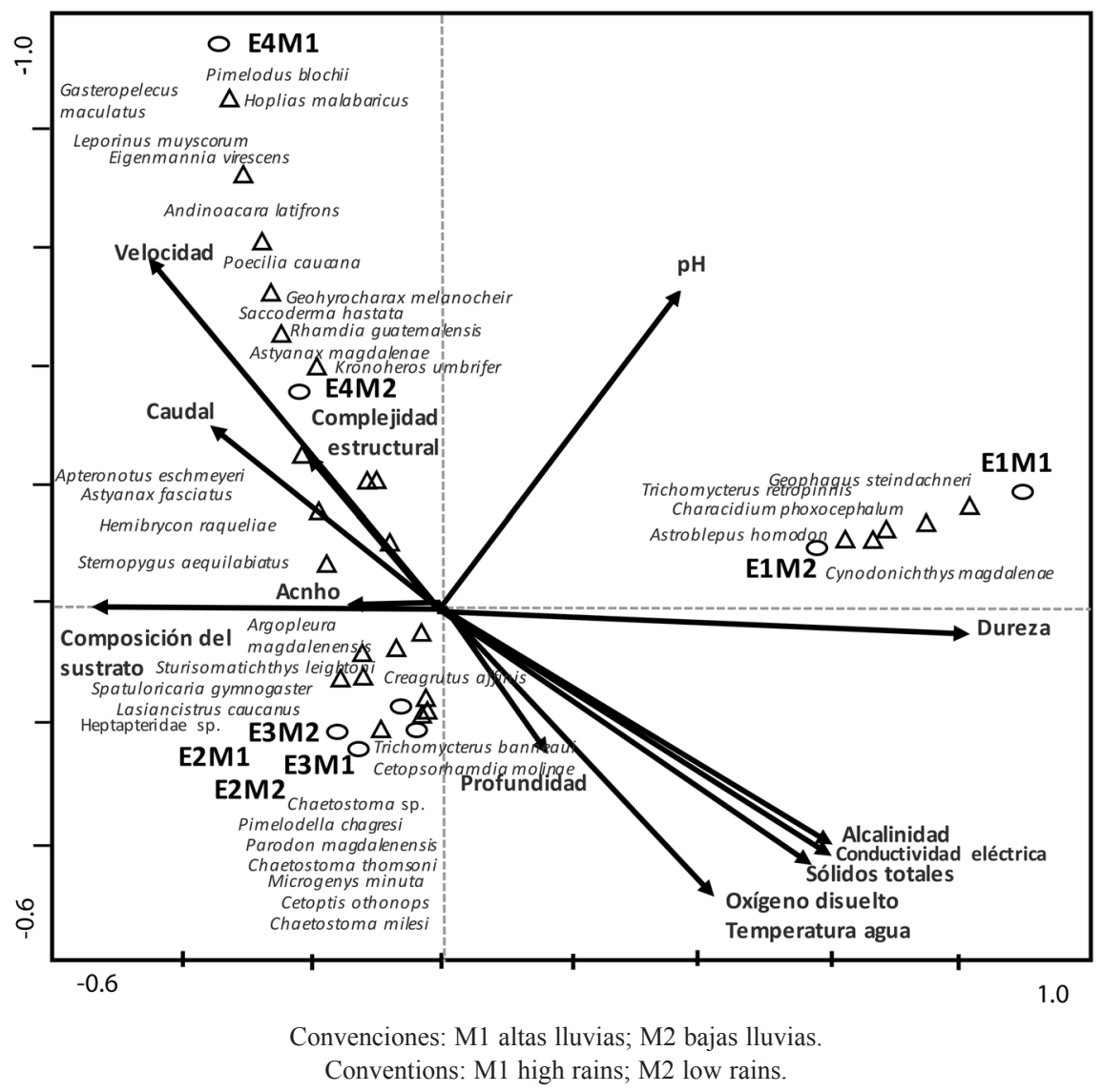

Fig. 3. Análisis de Correspondencia Canónica de las especies, variables ambientales y estaciones evaluadas en la microcuenca del río Anchique.

Fig. 3. Canonical Correspondence Analysis of the species, environmental variables and sampling points evaluated in the Anchique river sub-basin. 


\section{DISCUSIÓN}

La mayor representación de los órdenes Siluriformes y Characiformes encontrada en el presente estudio, es un patrón general no solo para la ictiofauna neotropical (Anderson \& Maldonado-Ocampo, 2010) sino también para la región del Alto Magdalena, en las microcuencas de los ríos Coello (Siluriformes 54.1 $\%$, Characiformes $34.2 \%$ ), Totare (Siluriformes $55 \%$, Characiformes $41 \%$ ), Opia (Characiformes $73.45 \%$, Siluriformes $22.1 \%$ ) y Alvarado (Siluriformes $52.8 \%$, Characiformes $35.3 \%$ ) (CORTOLIMA, 2004; CORTOLIMA, 2007; López-Delgado, 2013; Albornoz-Garzón \& Conde-Saldaña, 2014).

El número de especies identificadas (36 especies), representan el $27 \%$ de las especies reportadas para el Alto Magdalena (Villa-Navarro et al., 2006) y un $17 \%$ de la cuenca del río Magdalena (Barletta et al., 2010). Adicionalmente, el número de especies registradas en el río Anchique es igual a lo reportado en ríos de mayor envergadura como el río Alvarado (36 spp.) (Albornoz-Garzón \& Conde-Saldaña, 2014), y menor a lo reportado en el río Opia (38 spp.) (López-Delgado, 2013).

La comunidad íctica del río Anchique se ajustó al modelo de distribución serie logarítmica, el cual sugiere una comunidad con abundancias altamente desiguales (Hill \& Hammer, 1998; He \& Tang, 2008), además el modelo puede ser resultado de efectos de muestreo y efectos estocásticos o definitivos (Boswell \& Patil, 1971; Hughes, 1986). Esto se ve reflejado en un tramo del río antes de E3, donde el efecto de la extracción de agua para riego (CORTOLIMA, 2007) podría generar una disminución de recursos disponibles para los peces al intervenir en los patrones hidrológicos de la cuenca y modificar los atributos físicos del hábitat, lo que podría actuar como un efecto definitivo y generando cambios en la comunidad íctica (Whittaker, 1972; Bazzaz, 1975; Gray, 1981; Nummerlin \& Kaitala, 2004; He \& Tang, 2008; Martins-Dias \& Tejerina-Garro, 2010). Además, durante el periodo de muestreo se presentó un año atípico en el ciclo hidrológico, como efecto del fenómeno del Niño el cual actuó como un evento estocástico en la comunidad íctica del río Anchique.

Adicionalmente, el régimen pluvial bimodal del bs- $\mathrm{T}$ genera variaciones hidrológicas que se reflejarían en las comunidades de peces por la intermitencia en el flujo del agua, el ancho del río y conectividad entre la cabecera y los sectores medio y bajo (Winemiller, 1996; Herbert \& Gelwick, 2003). Sin embargo, los resultados del presente estudio no soportan esta teoría ya que no se observaron diferencias en la composición y estructura de la comunidad íctica entre las épocas evaluadas.

La inexistencia de variación temporal entre las comunidades de peces podría explicarse por la alteración en el régimen bimodal del primer semestre de 2014. En este orden, febrero se comportó como el segundo mes más lluvioso $($ media $=49.3 \mathrm{~mm}, 2014=73.5 \mathrm{~mm})$, y abril no fue el mes de mayor precipitación (media $=60.7 \mathrm{~mm}, 2014=49.8 \mathrm{~mm}$ ). Lo anterior sugiere un efecto de la escala de trabajo, ya que, la fuerte relación entre la estructura de las comunidades y el régimen hidrológico no siempre puede ser verificado a escalas temporales ecológicas y espaciales locales (Herbert \& Gelwick, 2003), por lo tanto, no se cumple a nivel temporal la hipótesis planteada en el presente estudio. Sin embargo, muchas especies de peces se mueven en respuesta a la variación espacial de un recurso disponible (Winemiller, 1989), lo que podría explicar las diferencias encontradas en las comunidades a nivel espacial a lo largo del río.

A lo anterior, es importante considerar que las características estructurales de los ambientes tropicales ejercen efectos en la composición de las comunidades ícticas (Mendoça, Magnuson, \& Zuanon, 2005), de tal forma que la heterogeneidad del hábitat, incluyendo variaciones en el sustrato (E1, E2 y E3 con fondos de roca, guijarro y arena, y E4 compuesto principalmente de hojarasca y lodo), los cuales influyen en los patrones de distribución de las comunidades de peces tropicales (Motta-Bührnheim \& Cox-Fernandes, 2003; Mueller \& Pyron, 2010). 
La persistencia de Creagrutus affinis y Trichomycterus banneaui podría explicarse por su capacidad de colonización de quebradas y ríos, así como la estrategia de reproducción continúa en C. affinis (Harold \& Vari, 1994), y a la presencia de odontodes en $T$. banneaui que le permiten resistir y ascender corrientes fuertes, colonizando hábitats con fondos de roca, guijarro y corrientes fuertes (García-Melo et al., 2005, Rodríguez, 2008).

Characidium phoxocephalum, C. affinis, Trichomycterus retropinnis, T. banneaui, Astroblepus homodon, Cynodonichthys magdalenae y Geophagus steindachneri conformaron la comunidad característica de la estación E1. Se destaca que esta estación se ubica en la cabecera del río y se caracteriza por registrar un escaso número de especies, de ahí el hecho de haber presentado la menor diversidad según los números efectivos de especies.

Según las teorías del Concepto del Río Continuo (Vannote et al., 1980) y del Pulso de inundación (Junk et al., 1989), en las cabeceras y zonas altas de los ríos se presenta menor diversidad y riqueza de especies, todo esto debido a cambios estacionales abruptos que se presentan en estas zonas, este patrón se observó en el río Anchique (E1) un río corto con un gradiente altitudinal no tan pronunciado. Este hecho se puede asociar a que el río no tiene tributarios que le aporten un mayor número de especies en su parte alta.

Adicionalmente, en E1 predominaron los fondos rocosos y arenosos, con perifiton, vegetación riparia arbórea, hojarasca y detritus, siendo un hábitat o ambiente adecuado para estas especies ícticas (Briñez-Vásquez et al., 2005; Castro-Roa et al., 2007), ya que, estos elementos sirven como sustrato para el desarrollo de alevines, zonas de alimentación, refugio y reproducción (Angermeier \& Karr, 1983), sin embargo, en E1 se encuentra una barrera (caída de agua) que limita el desplazamiento de las poblaciones de ictiofauna.

La riqueza local y la composición de las comunidades ícticas, tienden a incrementarse de forma directa con el ancho y volumen en los cuerpos de agua (Toepfer, Williams, Martínez,
\& Fisher, 1998; Grenouillet, Pont, \& Herisse, 2004). Los análisis multivariados como el ACC, son consistentes con el aumento en la complejidad estructural aguas abajo, generada por la presencia de vegetación riparia, así como hojarasca y raíces sumergidas (Mueller \& Pyron, 2010).

Lo anterior explicaría, en parte, la presencia $E$. virescens, S. aequilabiatus y $A$. eschmeyeri en la parte baja del río (E4), donde la vegetación enraizada sumergida puede ser utilizada para su refugio y alimentación (Ferreira \& Casatti, 2006; Barreto-Teresa \& Mei-Romero, 2010). Adicionalmente, E4 se encuentra ubicada más cerca a la desembocadura con el río Magdalena, lo cual facilita la migración de especies como L. muyscorum, S. leightoni y $P$. blochii al río Anchique, siendo uno de los factores que incrementa la diversidad en pequeños tributarios cercanos a grandes ríos (Gorman, 1986; Fernandes, Podos, \& Lundberg, 2004).

El uso de números efectivos permite una mejor interpretación de la diversidad de las comunidades a nivel espacial y temporal, de acuerdo a esto, E4 registro el valor más alto de riqueza $(0 \mathrm{D}=24 \mathrm{spp}$. $)$ y diversidad $\left({ }^{1} \mathrm{D}=\right.$ 14 spp.), lo cual se puede atribuir a la complejidad estructural del hábitat y composición del sustrato ofertado, estas características podrían ofertar más refugios y proporcionar condiciones óptimas para la colonización de un mayor número de especies ícticas (Cala, 2001). Asimismo, algunos componentes de la complejidad estructural como hojas y ramas sumergidas, sirven como sustrato para el desarrollo de microorganismos, proveen zonas de alimentación para macroinvertebrados acuáticos y peces, y ofrecen refugios contra depredadores a la ictiofauna (Angermeier \& Karr, 1983; Casatti et al., 2012).

Finalmente, la comunidad de peces de la microcuenca del río Anchique no presenta variación a nivel temporal, pero sí a nivel espacial, lo cual podría deberse a que cada estación de muestreo presentó características estructurales muy disimiles entre sí, como la complejidad estructural, composición del sustrato y el ancho del río. 


\section{AGRADECIMIENTOS}

A la Corporación Autónoma Regional del Tolima CORTOLIMA y la Oficina de Investigaciones y Desarrollo Científico de la Universidad del Tolima por la financiación del presente trabajo.

\section{RESUMEN}

La microcuenca del río Anchique se ubica en el municipio de Natagaima (Tolima) y drena directamente al río Magdalena, la distribución y abundancia de los peces está influenciada por múltiples factores a escalas temporales y espaciales, el objetivo del estudio es describir la estructura y composición de la comunidad íctica a lo largo del río y probar la hipótesis de que estas comunidades varían a nivel temporal y espacial. Se realizaron dos muestreos en altas y bajas lluvias, y se establecieron cuatro estaciones, colectándose 625 ejemplares y 36 especies. No se encontraron diferencias estadísticamente significativas entre algunas variables ambientales del río a nivel temporal, según la prueba Kruskal-wallis. Los valores de los índices de complejidad estructural y composición del sustrato, muestran que la estación E4 presentó los valores más altos, además fue la estación con mayor riqueza y diversidad según los números de Hill. El análisis de correspondencia canónica evidenció que el sustrato, complejidad y ancho del cauce influyeron de forma significativa en la composición y distribución de los peces. La comunidad íctica del río no presentó variación de la diversidad y riqueza temporalmente, pero si espacialmente, lo cual podría deberse a que cada estación presentó características estructurales diferentes.

Palabras clave: Ictiofauna, ecosistema acuático, variables ambientales, río Anchique.

\section{REFERENCIAS}

Albornoz-Garzón, J. G., \& Conde-Saldaña, C. C. (2014). Diversidad y relaciones ecomorfológicas de la comunidad íctica de la cuenca del rio Alvarado, Tolima, Colombia (Tesis de pregrado). Universidad del Tolima, Ibagué, Colombia.

Anderson, E. P., \& Maldonado-Ocampo, J. A. (2010). A regional perspective on the diversity and conservation of tropical Andean fishes. Conservation Biology, 25(1), 30-39. doi:10.1111/j.1523-1739.2010.01568.x

Angermeier, P. L., \& Karr, J. R. (1983). Fish communities along environmental gradients in a System of Tropical streams. Environmental Biology of Fishes, 9(2), 117-135.

Angermeier, P. L., \& Karr, J. R. (1984). Relationships between woody debris and fish habitat in a small waemwater stream. Transactions of the American Fisheries society, 6(113), 716-726.

Barletta, M., Jaureguizar, A., Baigun, C., Fontoura, N. F., Agostnho, A. A., Almeida-Val, V. M. F.,... Correâ, M. F. M. (2010). Fish and aquatic habitat conservation in South America: a continental overview with emphasis on neotropical systems. Journal of Fish Biology, 76(9), 2118-2176. doi:10.1111/j.1095-8649.2010.02684.x

Barreto-Teresa, F., \& Mei-Romero, R. (2010). Influence of the riparian zone phytophysiognomies on the longitudinal distribution of fishes: evidence from a Brazilian savanna stream. Neotropical Ichthyology, 8(1), 163-170.

Bazzaz, F. A. (1975). Plant species diversity in old-field successional ecosystems in southern Illiniois. Ecology, 56(2), 485-488. doi: 10.1007/BF00877430

Boswell, M. T. and Patil, G. P. (1971).Chance mechanisms generating logarithmic series distribution used in the analysis of number of species and individuals. Statistical Ecology, 1, 99-130.

Braak, C. J., Ter, F., \& Smilauer, P. (2009). Canoco. Wageningen, The Netherlands: Biometris Plant Research International.

Briñez-Vásquez, G. N., Villa-Navarro, F. A., Ortega-Lara, A., Reinoso-Flórez, G., \& García-Melo, J. G. (2005). Distribución altitudinal y diversidad de la familia Astroblepidae (Pisces, Siluriformes), en la cuenca del río Coello, Tolima. Dahlia, 2005(8), 39-46.

Buitrago, U. A. (1995). Sistemática de las especies colombianas del género Astroblepus Humboldt 1805 (Pisces: Siluroidei: Astroblepidae) (Tesis de maestría). Universidad Nacional de Colombia, Bogotá, Colombia.

Bussing, W. A., \& López, M. I. (1977). Distribución y aspectos ecológicos de los peces de las cuencas hidrográficas de Arenal, Bebedero y Tempisque, Costa Rica. Revista Biología Tropical, 25(1), 13-37.

Cala, P. (2001). Ictiofauna Epicontinental de Colombia en el contexto global Neotropical y su estado actual: una revisión bibliográfica. Dahlia, 2001(4), 3-14.

Casatti, L., Barreto-Teresa, F., Gonçalves-Souza, T., Bessa, E., Manzotti, A., R., da Silva Gonçalves, C., \& de Oliveira-Zeni, J. (2012). From forests to cattail: how does the riparian zone influence stream fish? Neotropical Ichthyology, 10(1), 205-214.

Castro-Roa, D., Villa-Navarro. F. A., García-Melo, J. E., García-Melo, L. J., Herrada-Yara, M. E., \& ReinosoFlórez, G. (2007). Distribución y aspectos biológicos de Bryconamericus tolimae en la cuenca del río Prado, Colombia. Dahlia, 2007(9), 77-86.

Chao, A., Gotelli, N. J., Hsieh, T. C., Sander, E. L., Ma, K. H., Colwell, R. K., \& Ellison, A. M. (2014). 
Rarefaction and extrapolation with Hill numbers: a framework for sampling and estimation in species diversity studies. Ecological Monographs, 84(1), 45-67.

Chapman, M. G., \& Underwood, A. J. (1999). Ecological patterns in multivariate assemblages: information and interpretation of negative values in ANOSIM tests. Marine Ecology Progress Series, 1999(180), 257-265.

Colwell, R. K. (2009). EstimateS: Statistical estimation of species richness and shared species from samples. Version 9 and earlier. User's Guide and application.

Corporación Autónoma Regional del Tolima (CORTOLIMA). (2004). Biodiversidad faunística de la cuenca del rio Coello, Biodiversidad regional fase I. Colombia: CORTOLIMA. Recuperado de https://www. cortolima.gov.co/

Corporación Autónoma Regional del Tolima (CORTOLIMA). (2007). Biodiversidad faunística de la cuenca del río Totare, Biodiversidad regional fase III. Colombia: CORTOLIMA. Recuperado de https:// www.cortolima.gov.co/

Corporación Autónoma Regional del Tolima "CORTOLIMA". (2009). Plan de Acción Trienal 2007-2009. Colombia: CORTOLIMA. Recuperado de http:// www.cortolima.gov.co/

Dahl, G. (1971). Los Peces del Norte de Colombia. Bogotá, Colombia: INDERENA.

De Santana, C. D., \& Maldonado-Ocampo, J. A. (2005). Apteronotus milesi, new species of ghost knifefish (Gymnotiformes: Apteronotidae) from the Cauca River, with a key to apteronotids from the Magdalena-Cauca basin, Colombia. Ichthyological Exploration of Freshwaters, 16(3), 223-230.

Eigenmann, C. (1922). The fishes of the Northwestern South America, part I. The fresh-water fishes of Northwestern South America, including Colombia, Panamá, and Pacific slopes of Ecuador and Peru, together with an appendix upon the fishes of the río Meta in Colombia. Memoirs of the Carnegie Museum, 9(1), 1-346.

Fernandes, C. C., Podos, J., \& Lundberg, J. G. (2004). Amazonian ecology: tributaries enhance the diversity of electric fishes. Science, 305(5692), 1960-1962.

Ferreira, C. P., \& Casatti, L. (2006). Influência da estrutura do habitat sobre e ictiofauna de um riacho emu ma micro-bacia de pastagem, São Paulo, Brasil. Revista Brasileira de Zoologia, 23(3), 642-651.

Ferreira, K. M. (2007). Biology and ecomorphology of stream fishes from the rio Mogi-Guacu basin, Southeastern Brazil. Neotropical Ichthyology, 5(3), 311-326.
Frissell, C. A., Liss, W. J., Warren, C. E., \& Hurley, M. D. (1986). A hierarchical framework for stream habitat classification: viewing streams in a watershed context. Environmental Management, 10(2), 199-214.

García-Melo, L., Villa-Navarro, F. A., Reinoso-Flórez, G., \& Ortega-Lara, A. (2005). Aspectos bioecológicos de los peces del género Trichomycterus (Siluriformes: Trichomycteridae) en la cuenca del río Coello, Colombia. Dahlia, 2005(8), 53-59.

Guerrero-Kommritz, J. (1997). Ensayos sobre pesca eléctrica en Colombia. Dahlia, 1997(2), 71-77.

Gorman, O. T. (1986). Assemblage organization of stream fishes: the effect of rivers on adventitious streams. The American Naturalist, 128(4), 611-616.

Gray, J. S. (1981). The Ecology of Marine Sediments. Oxfordshire, England: Oxford University Press.

Greenberg, L. A. (1991). Habitat use and feeding behavior of thirteen species of benthic stream fishes. Environmental Biology of Fishes, 31(4), 389-401. doi: 10.1007/BF00002364

Grenouillet, G., Pont, D., \& Herisse, C. (2004). Within basin fish assemblage structure: the relative influence of habitat versus stream spatial position on local species richness. Canadian Journal of Fisheries and Aquatic Sciences, 61(1), 93-102. doi: 10.1139/ f03-145

Harding, J. M., Burky, A. J., \& Way, C. M. (1998). Habitat preferences of the rainbow darter, Etheostoma coeruleum, with regard to microhabitat velocity shelters. Copeia, 1998(4), 988-997.

Harold, A. S., \& Vari, R. P. (1994). Systematics of the Trans-Andean Species of Creagrutus (Ostariophysi: Characiformes: Characidae). Smithsonian Contributions to Zoology, 551.

He, F., \& Tang, D. (2008). Estimating the niche preemption parameter of the geometric series. Acta Ecologica, 33(2008), 105-107. doi:10.1016/j.actao.2007.10.001

Herbert, M. E., \& Gelwick, F. P. (2003). Spatial variation of headwater fish assemblages explained by hydrologic variability and upstream effects of impoundement. Copeia, 2003(2), 273-284. doi:http://dx.doi. org/10.1643/0045-8511(2003)003[0273:SVOHFA]2 $.0 . \mathrm{CO} ; 2$

Hill, M. O. (1973). Diversity and evenness: a unifying notation and its consequences. Ecology, 54(2), 427-432.

Hill, K., \& Hammer, K. C. (1998). Using species abundance models as indicator of habitat disturbance in tropical forests. Journal of Applied Ecology, 1998(35), 458-460.

Hughes, R. G. (1986). Theories and models of species abundance. The American Naturalist, 128(6), 879-899. 
Jost, L. (2006). Entropy and diversity. Oikos, 113(2), 363-375.

Junk, W. J., Bayley, P. B., \& Sparks, E. (1989). The Flood Pulse Concept in River-Floodplain Systems. Canadian Journal of Fisheries and Aquatic Sciences, 1(106), 110-127.

López-Delgado, E. O. (2013). Composición y estructura de la comunidad de peces y sus relaciones con la calidad de la vegetación riparia y algunas variables ambientales en dos ríos de bosque seco tropical (bs-t), Tolima (Colombia) (Tesis de maestría). Universidad del Tolima, Ibagué, Colombia.

Magurran, A. E. (2004). Measuring Biological Diversity. Oxfordshire, England: Blackwell Publishing.

Maldonado-Ocampo, J., Ortega-Lara, A., Usma-Oviedo, J. S., Galvis-Vergara, G., Villa-Navarro, F. A., VásquezGambona, L., Prada-Pedreros, S., \& Ardila C. (Ed) (2005). Peces de los Andes de Colombia. Bogotá, Colombia: Instituto de Investigación de Recursos Biológicos Alexander von Humboldt.

Martins-Dias, A., \& Tejerina-Garro, F. L. (2010). Changes in the structure of fish assemblages in streams along an undisturbed-impacted gradient, upper Paraná River basin, Central Brazil. Neotropical Ichthyology, $8(3), 587-598$

Martin-Smith, K. M. (1998). Relationships between fishes and habitat in rainforest streams in Sabah, Malaysia. Journal of Fish Biology, 52(3), 458-482. doi:10.1111/j.1095-8649.1998.tb02010.x

Mendoça, F. P., Magnuson, W. E., \& Zuanon, J. (2005) Relationships between habitat characteristics and fish assemblages in small streams of Central Amazonia. Copeia, 2005(4), 751-764.

McGill, B. J., Etienne, R. S., Gray, J. S., Alonso, D., Anderson, M. J., Benecha, H. K., \& Dornelas, M. (2007). Species abundance distributions: moving beyond single prediction theories to integration within an ecological framework. Ecology letters, 10(10), 9951015. doi:10.1111/j.1461-0248.2007.01094.x

Moreno, C. (2001). Métodos para medir la biodiversidad. Hidalgo, México: GORFI S.A.

Motta-Bührheim, C., \& Cox-Fernandes, C. (2003). Structure of fish assemblages in amazonian rain-forest streams: effects of habitats and locality. Copeia, 2003(2), 255-262.

Mueller, R. J., \& Pyron, M. (2010). Fish assemblages and substrates in the middle Wabash River, USA. Copeia, 2010(1), 47-53.

Nummerlin, M., \& Kaitala, S. (2004). Do species dominance indices indicate rain forest disturbance by logging. Biotropica, 36(4), 628-632.
Oliveira, R. E. M. C. C., \& Pessanha, A. L. M. (2014). Fish assemblages along a morphodynamic continuum on three tropical beaches. Neotropical Ichthyology, 12(1), 165-175.

Power, M. E., Sun, A., Parker, M., Dietrich, W. E., \& Wootton, J. T. (1995). Hydraulic food-chain

models: an approach to the study of foodweb dynamics in large rivers. BioScience 45(3), 159-167.

R Development Core Team. (2016). R: a language and environment for statistical computing. R Foundation for Statistical Computing, Vienna.

Resh, V. H., Brown, A. V., Covich, A. P., Gurtz, M. E., Li, H. W., Minshall, G. W., ... Wissmar, R. C. (1988). The Role of Disturbance in Stream Ecology. Journal of the North American Benthological Society, 7(4), 433-455.

Rodríguez, C. A. A. (2008). Trichomycterus cachiraensis (Siluriformes: Trichomycteridae), nueva especie del rio Cachira. Dahlia, 2008(10), 33-41.

Sousa, W. P. (1984). The role of disturbance in natural communities. Annual Review of Ecology and Systematics, 15(1984), 353-391.

Stewart, D. J., Ibarra, M., \& Barriga-Salazar, R. (2002). Comparison of deep-river and adjacent sandy-beach fish assemblages in the Napo River basin, eastern Ecuador. Copeia, 2002(2), 333-343.

Toepfer, A. S., Williams, L. R., Martínez, A. D., \& Fisher, W. L. (1998). Fish and Habitat Heterogeneity in Four Streams in the Central Oklahoma/Texas Plains Ecoregion. Academy of Sciences, 1998(78), 41-48.

Vannote, R. L., Minshall, G. W., Cummins, K. W., Sedell, J. R., \& Cushing, C. F. (1980). The River Continuum Concept. Canadian Journal of Fisheries and Aquatic Sciences, 37(1), 130-137.

Villa-Navarro, F. A., \& Losada-Prado, S. (1999). Aspectos tróficos de Petenia umbrifera (Pisces: Cichlidae) en la represa de Prado (Tolima). Revista de la Asociación Colombiana de Ciencias Biológicas, 11(1), 24-35.

Villa-Navarro, F., Zúñiga-Upegui, P. T., Castro-Roa, D., García Melo, L., García, J., \& Herrada, M. (2006). Peces del alto Magdalena, cuenca del río Magdalena, Colombia. Biota Colombiana, 7(1), 3-22.

Villa-Navarro, F. A., \& Losada-Prado, S. (2004). Aspectos bioecológicos del caloche, Sternopygus macrurus (Gymnotiformes: Sternopygidae), en la represa de Prado, Tolima, Colombia. Dahlia, 2004(7), 49-56.

Whittaker, R. H. (1972). Evolution and Measurement of Species Diversity. Taxon, 21(2/3), 213-251. 
Winemiller, K. O. (1989). Patterns of variation in life history among South American fishes in seasonal environments. Oecologia, 81(2), 225-241.

Winemiller, K. O. (1996). Dynamic diversity in fish assemblages of tropical rivers. Texas, USA: Academic Press Inc.

Winemiller, K. O., López-Fernández, H., Taphorn, D. C., Nico, L. G., \& Duque, A. B. (2008). Fish assemblages of the Casiquiare river, a corridor and zoogeographical filter for dispersal between the Orinoco and
Amazon basins. Journal of Biogeography, 35, 15511563. doi:10.1111/j.1365-2699.2008.01917.x

Zaret, T. M., \& Rand, A. S. (1971). Competition in tropical stream fishes: support for the competitive exclusion principle. Ecology, 52(2), 336-342.

Zúñiga-Upegui, P. T., Villa-Navarro, F. A., Ortega-Lara, A., \& Reinoso-Flórez, G. (2005). Relación longitudpeso y frecuencias de tallas para los peces del género Chaetostoma (Siluriformes, Loricariidae) de la cuenca del río Coello, Colombia. Dahlia, 8, 47-52. 